\title{
Sehr geehrte Damen und Herren, liebe Kolleginnen und Kollegen,
}

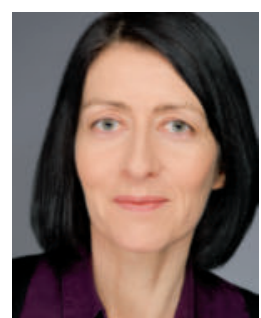

Prof. Dr. Kirsten Minden auch in diesem Jahr nimmt sich die Zeitschrift „arthritis + rheuma“ wieder kinder- und jugendrheumatologischen Themen in einer entsprechenden Rubrik an.

Der Fokus der Übersichtsbeiträge wird auf Komorbiditäten bei rheumatischen Erkrankungen mit Beginn im Kindes- und Jugendalter liegen. Das Spektrum möglicher krankheits- und/oder therapieassoziierter Manifestationen an Organen/Organsystemen ist breit, die Beiträge werden das veranschaulichen.

Um psychische Begleiterkrankungen geht es in diesem Heft. Diese beginnen in etwa drei Viertel aller Fälle im Jugend- und jungen Erwachsenenalter, junge Menschen mit einer chronischen Erkrankung haben ein besonders hohes Risiko für psychische Belastungen wie Angst und Depression. Die Häufigkeit psychischer Begleiterkrankungen bei Jugendlichen mit einer juvenilen idiopathischen Arthritis (JIA) wird mit bis zu 35 \% angegeben, worunter depressive Störungen dominieren. Deren frühzeitige Erkennung ist notwendig, um Betroffenen rechtzeitig zusätzliche Unterstützung anbieten zu können. Geschieht das nicht, werden Möglichkeiten, die Langzeitprognose und Lebensqualität der Betroffenen zu verbessern, verpasst. Psychische Belastungen werden in der klinischen Praxis oft nur am Rande berücksichtigt. Nach Daten des Biologika-Registers JuMBO ist mindestens jeder zehnte junge Rheumatiker von einer Depression betroffen, bei nicht einmal jedem zweiten Betroffenen hat der Rheumatologe auch Kenntnis davon. Wie groß das Problem unter den aktuell von uns betreuten Jugendlichen ist, wird in diesem Jahr im Rahmen des interdisziplinären Forschungsverbundes $\mathrm{COACH}$ (Chronic Conditions in Adolescents: Implementation and Evaluation of Patient-centred Collaborative Healthcare) untersucht, der ebenfalls im Beitrag vorgestellt wird.

Neben der Uveitis und psychischen Erkrankungen ist der Hypertonus eine der häufigsten Begleiterkrankungen bei jungen Rheumatikern. Er stellt einen Risikofaktor für Herz-Kreislauferkrankungen dar, die bei Erwachsenen mit entzündlich rheumatischen Erkrankungen signifikant häufiger als in der Bevölkerung vorkommen und zur verkürzten Lebenserwartung der Patienten beitragen. Was über das Risiko hinsichtlich Herz-Kreislauferkrankungen bei juvenilen rheumatischen Erkrankungen bekannt ist, darüber wird Herr Dr. Milatz in der kommenden Ausgabe 2/2019 berichten.

Begleitende Nierenerkrankungen sind bei Patienten mit rheumatischen Erkrankungen durch die frühzeitigeren Therapien mit weniger nephrotoxischen Substanzen und besserer Entzündungskontrolle seltener geworden. Wie groß das Risiko einer First-line-Therapie der JIA mit nichtsteroidalen Antirheumatika dennoch ist, wird in Ausgabe 3/2019 aus nephrologischer Sicht dargestellt.

Im Unterschied zu den inneren Organen führt uns die Haut Probleme unmittelbar vor Augen. Hautveränderungen können krankheitsunabhängig auftreten, für die rheumatische Erkrankung diagnosegebend sein, im Sinne von „Alarmzeichen“ auf Erkrankungsschübe hinweisen oder auch Medikamentenunverträglichkeiten anzeigen. Frau Priv.-Doz. Dr. Vogt wird im Heft 4/2019 auf klinisch relevante Befunde eingehen.

Krankheits- und/oder therapiebedingt haben Kinder und Jugendliche mit rheumatischen Erkrankungen ein höheres Risiko für Infektionen, was in Heft 5/2019 thematisiert wird. Wirksame präventive Maßnahmen stehen mit Impfungen zur Verfügung. In diesem Jahr dürfen wir die neuen STIKO-Empfehlungen zu Impfungen bei Autoimmunerkrankungen und unter immunmodulatorischer Therapie erwarten.

Abgerundet wird die Themenserie mit Augenmanifestationen bei rheumatischen Erkrankungen, wobei die Uveitis die häufigste extraartikuläre Manifestation bei der JIA ist. Diese stand in den vergangenen Monaten wiederholt im Blickpunkt, deshalb wird es um Manifestationen jenseits der JIA-assoziierten Uveitis gehen. 
Neben den Übersichtsbeiträgen erwarten Sie auch im Jahr 2019 interessante Kasuistiken. Einen spannenden Fall einer lgG4-assoziierten Aortitis bei einem 6-jährigen Jungen stellen Ihnen die Kollegen Youssef, Lutz und Helling-Bakki aus Heidelberg in diesem Heft vor.

Ich hoffe, dass Ihnen die Auswahl der Themen zusagt und Sie Freude beim Lesen haben. Den Autorinnen und Autoren der Übersichtsbeiträge und Kasuistiken sei an dieser Stelle ganz herzlich für die Unterstützung bei der Gestaltung und den Herausgebern der „arthritis + rheuma“ bei der Umsetzung der kinderrheumatologischen Rubrik gedankt.

Ihnen allen wünsche ich ein gesundes und erfolgreiches neues Jahr.

Ihre

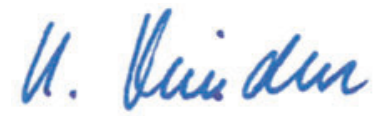

Kirsten Minden

Berlin

Schriftleitung Kinderrheumatologie 